\title{
Sobrevivencia y crecimiento de plántulas reforestadas de Podocarpus glomeratus (Podocarpaceae) en diferentes altitudes y micrositios en ecosistemas de pastizales de los Andes bolivianos después de cuatro años
}

\author{
Ariel Isaías Ayma-Romay ${ }^{1, \varpi} ;$ Pedro Lovera $^{1,3}$, \& Gladys Soto-Rojas ${ }^{1,2}$ \\ ${ }^{1}$ Proyecto de Manejo y Restauración de Bosques en Independencia - Fundación para la Autogestión del Medio Ambiente \\ (FUPAGEMA). Cochabamba, Bolivia. ${ }^{2}$ Universidad Mayor de San Simón, Escuela de Ciencias Forestales. Cochabamba, \\ Bolivia. ${ }^{3}$ Gobierno Autónomo Municipal de Independencia. Cochabamba, Bolivia.
}

\begin{abstract}
Resumen. La altitud y el micrositio son factores clave para el establecimiento de las plantas en ecosistemas de montaña. En este estudio evaluamos cómo la sobrevivencia y el crecimiento de plántulas de Podocarpus glomeratus, una conífera nativa vulnerable a la extinción, varía a través de ambos factores. Plantamos 150 plántulas de la especie, repartidas en 15 parcelas a lo largo de un gradiente altitudinal con cinco altitudes (2700, 3000, 3300, 3600 y 3900 m s.n.m) y tres repeticiones por altitud (parcelas anidadas) en ecosistemas de pastizales de la comunidad de Pajchanti (Cochabamba, Bolivia). La sobrevivencia, crecimiento y los componentes del micrositio alrededor de cada plántula (porcentaje de cobertura de pastos, arbustos, piedras, musgos, hierbas y porcentaje de pendiente) se evaluaron tres veces durante cuatro años. La influencia de la altitud sobre la sobrevivencia final o el crecimiento acumulado de cada plántula fueron analizados con modelos lineales generalizados mixtos (MLGM) y pruebas de Tukey (incluyendo a las parcelas repetidas como factor aleatorio). Asimismo, la influencia de cada uno de los componentes del micrositio sobre la sobrevivencia o el crecimiento de cada plántula fueron analizados por separado para cada altitud, también con MLGM, para que los efectos de esas variables no se confundan debido a su correlación. La sobrevivencia de las plántulas fue mayor en la altitud intermedia y menor en ambos extremos altitudinales. Sin embargo, el crecimiento fue mayor en el rango de la altitud inferior y la intermedia (2700 a 3300 m s.n.m) y menor en altitudes mayores. Los componentes del micrositio no influyeron en la sobrevivencia. Sin embargo, la cobertura de arbustos incrementó el crecimiento en la altitud inferior, la cobertura de piedras redujo el crecimiento en la altitud intermedia, y mayor porcentaje de pendiente y cobertura de piedras incrementaron el crecimiento a $3590 \mathrm{~m}$ s.n.m. La reforestación de $P$. glomeratus en ecosistemas de pastizales debería realizarse entre 2700 y 3300 m s.n.m. Sin embargo, las piedras, arbustos y pendiente, dependiendo su porcentaje de cobertura en el micrositio e interacciones con la altitud, incrementan o reducen el establecimiento de las plántulas, por lo que deben manejarse en futuras reforestaciones.
\end{abstract}

[Palabras clave: altura, reforestación, montaña, facilitación]

\begin{abstract}
Aвstract. Seedling survival and growth of Podocarpus glomeratus (Podocarpaceae) along different altitudes and microsites in grassland ecosystems from the central Andes of Bolivia after four years. Altitude and microsite are key factors for the establishment of seedlings in mountain ecosystems. In this study, we evaluated how seedling survival and growth of Podocarpus glomeratus, a native tree vulnerable to extinction, varied across different altitudes and microsites. As part of a four-year reforestation program, 150 seedlings were planted in 15 experimental plots along an altitudinal gradient of five levels $(2747,3005,3300,3590$ and $3850 \mathrm{~m}$ a.s.l.) and three repetitions by altitude (nested plots) in a grassland ecosystems from the community of Pajchanti (Cochabamba, Bolivia). Plant survival, growth and microsite around each seedling, such as slope and ground cover (rock outcrops, grasses, shrubs, mosses and herbs) were measured three times during the reforestation program. Generalized linear mixed models (GLMM) and Tukey tests were used in order to evaluate the influence of altitude on seedling survival and growth (including nested plots as random factor). The influence of each component biotic or abiotic in the microsites on the survival and growth was analyzed also with GLMM, for each altitude separately. Thus, we avoided confusing effect of altitude and microsite. Seedlings survival was significantly greater at the intermediate altitude and lower at both altitudinal extremes, but seedlings growth was greater at the lower altitude range (from 2700 to $3300 \mathrm{~m}$ a.s.l.), while smaller at higher altitudes. Seedlings survival was not influenced by abiotic or biotic components of the microsite. However, high shrub cover was related to higher seedlings growth at the lowest altitude. High rock outcrops cover had a negative effect on growth in the intermediate altitude, while more rock outcrops and higher slope percentage had positive effect on the growth at a higher altitude (3590 $\mathrm{m}$ a.s.l.). Reforestation of $P$. glomeratus in grassland ecosystems should be carried out at 2700 to $3300 \mathrm{~m}$ a.s.l. Nonetheless, shrubs, rock outcrops and slope of the microsite influenced the establishment of the seedlings, depending on cover percentage and interactions with the altitude. These microsite components must be managed in future reforestations.
\end{abstract}

[Keywords: forestation, mountain, facilitation]

Editora asociada: Ana Cingolani

ariel.isaias.aymar@gmail.com
Recibido: 30 de noviembre de 2015

Aceptado: 7 de febrero de 2017 


\section{INTRODUCCIÓN}

La reforestación en ecosistemas de pastizales está limitada por la elevada mortalidad y reducida tasa de crecimiento de las plántulas arbóreas nativas (Renison et al. 2004; Doust et al. 2006). En las montañas, la altitud y el micrositio son factores clave que controlan el establecimiento de las plántulas arbóreas en las prácticas de reforestación. Por ejemplo, la altitud impone las condiciones climáticas al marcar límites altitudinales óptimos y marginales (Körner and Paulsen 2004; Anthelme et al. 2014a), y el micrositio define en una escala menor las condiciones microambientales alrededor de las plántulas (Grubb 1977).

En la actualidad es dificultoso detectar las altitudes y los micrositios adecuados para reforestar árboles nativos porque las condiciones ambientales para las plantas están cambiando debido al calentamiento global (Anthelme et al. 2014a) y al cambio de uso del suelo (Báez et al. 2011; Marcora et al. 2013). Por ejemplo, se ha propuesto que en los Andes tropicales, el incremento de la temperatura será de $4.5^{\circ} \mathrm{C}$ hasta fin de siglo. Este cambio puede desplazar hacia altitudes mayores a las especies arbóreas adaptadas al frío (Beniston 2003; Báez et al. 2011). Asimismo, la deforestación y la degradación del suelo están formando extensos pastizales con alta exposición a la radiación solar, temperaturas extremas y déficit hídrico en un amplio rango de altitudes, lo cual dificulta aun más el establecimiento de plántulas arbóreas (Renison et al. 2004; Marcora et al. 2013). Estos cambios reducen la posibilidad de que las plántulas se establezcan en las mismas altitudes en las cuales se establecieron los árboles maduros.

Ante esta situación, identificar las altitudes y los micrositios adecuados en ecosistemas de pastizales puede contribuir al establecimiento de plántulas arbóreas. Así se puede manejar las condiciones ambientales impuestas por la altitud (Körner and Paulsen 2004), los efectos del calentamiento global (Anthelme et al. 2014a) y el estrés ambiental causado por la degradación del suelo y de la vegetación (Bertness and Callaway 1994). Por ejemplo, algunos estudios muestran que las plántulas de árboles nativos pueden tener mejor sobrevivencia o crecimiento en altitudes mayores o menores de la distribución de los bosques maduros (Cierjaks et al. 2007; Báez et al. 2011; Marcora et al. 2013). Asimismo, algunos componentes bióticos o abióticos alrededor de las plántulas arbóreas, como la cobertura de musgos, hierbas, pastos o arbustos (Zamora et al. 2004; Padilla and Pugnaire 2006), mayores pendientes (Simpson 1986; Fjeldså and Kessler 2004) y la presencia de piedras (Peters et al. 2008) pueden modificar el efecto de la altitud y de la degradación; esto facilita el establecimiento de las plántulas (Báez et al. 2011), debido a que pueden reducir el estrés ambiental causado por la alta radiación solar, la elevada evaporación en el suelo y las oscilaciones de temperatura, entre otros (Padilla and Pugnaire 2006).

En Bolivia, los bosques dominados por la conífera nativa de pino de monte (Podocarpus glomeratus D. Don) han sido fuertemente deforestados y degradados a pastizales (Ayma-Romay et al. 2007). Actualmente forman pequeños fragmentos de bosque y son vulnerables a la extinción (Navarro 2005) en las altitudes más altas observadas para el género Podocarpus en Sudamérica (2500 a 3500 m s.n.m.) (Dalling et al. 2011). Aún se comprende poco acerca del efecto de la altitud y el micrositio sobre el establecimiento de las plántulas arbóreas, y en particular de esta especie. En la actualidad, las reforestaciones con $P$. glomeratus se realizan en las mismas altitudes en las que se encuentran los bosques maduroscon pocoéxito(observación personal), pero se desconoce si sus plántulas se pueden establecer hacia altitudes mayores o menores (aunque se ha sugerido que las especies del género son sensibles al calentamiento global y a la sequía (Cernusak et al. 2011) y, por tanto, deberían desplazarse hacia altitudes mayores (Beniston 2003).

Por otra parte, también se desconoce el efecto de algunos componentes bióticos o abióticos en los pastizales, que podrían formar micrositios adecuados para las plántulas. Algunos estudios sugieren que las plántulas del género Podocarpus requieren micrositios que reduzcan la radiación solar y el déficit hídrico, dado que son tolerantes a la sombra (Cernusak et al. 2011) y sensibles a la deshidratación (Dalling et al. 2011). Por eso, su establecimiento es mayor en claros de bosque maduro e insignificante afuera del bosque (Ayma-Romay and Padilla 2009).

La hipótesis de este estudio es que las plántulas reforestadas de $P$.glomeratus pueden sobrevivir y crecer en un rango amplio de altitudes, facilitadas por algunos componentes abióticos o bióticos del micrositio. El objetivo 
general del trabajo fue comprender el efecto de la altitud y los componentes bióticos y abióticos del micrositio sobre la sobrevivencia y el crecimiento de plántulas de $P$. glomeratus reforestadas en pastizales de la comunidad de Pajchanti (Cochabamba, Bolivia) después de cuatro años.

\section{Materiales y MÉTOdos}

\section{Área de estudio}

La comunidad de Pajchanti se encuentra en el municipio de Independencia, provincia Ayopaya, al noroeste de Cochabamba, Bolivia $\left(17^{\circ} 5^{\prime} \mathrm{S}-66^{\circ} 49^{\prime} \mathrm{O}\right)$. El área tiene diferentes condiciones climáticas y de vegetación entre 2500 y 4200 m s.n.m y pertenece a los yungas bolivianos (distrito Yungas del Cotacajes), con transición o contacto con la puna peruana (distrito del Tunari) (Navarro 2005). El bioclima es pluviestacional y cambia de mesotropical a orotropical con el incremento de la altitud. A 2500 m s.n.m, la temperatura media anual es $14{ }^{\circ} \mathrm{C}$ y la precipitación promedio anual es $569 \mathrm{~mm}$, mientras que a 4200 m s.n.m, la temperatura media anual es $7.4^{\circ} \mathrm{C}$ y la precipitación promedio anual es 894 $\mathrm{mm}$ (Navarro 2005). Actualmente, los bosques maduros y de sucesión de $P$. glomeratus se encuentran en el piso altimontano entre 3000 y 3500 m s.n.m., alrededor de un ecosistema agrícola y de pastizales resultado de la deforestación para la agricultura y ganadería, entre el año 1950 y 1990. Los pastizales están dominados por pastos de Festucadolichophylla y Stipa ichu y arbustos dispersos del género Baccharis, Berberis y Miconia (Ayma-Romay and Padilla 2009). Estos pastizales se extienden hacia más abajo hasta los 2500 m s.n.m. y más arriba hasta los 4200 m s.n.m.

\section{Experimento}

Se instalaron 15 parcelas de $17 \times 17 \mathrm{~m}$, clausuradas con alambre de púas. Estas parcelas se repartieron en cinco altitudes (2747, 3005, 3300, 3590 y 3805 m s.n.m.) y se repitieron tres veces en cada altitud, con una distancia entre ellas de 500 a 1000 m. La reforestación se realizó con 150 plántulas de P. glomeratus de $5.3 \mathrm{~cm}$ de altura (mínimo $=2.5$ $\mathrm{cm}$; máximo $=10 \mathrm{~cm}$ ), extraídas de diferentes partes del bosque a una altitud de $3100 \pm 100$ m s.n.m. y plantadas en menos de cinco días. Las plántulas se extrajeron del bosque porque la producción de plántulas en vivero es difícil dada su producción de semillas esporádica y asincrónica (Ayma-Romay and Sanzetenea 2008). Además, porque en el bosque secundario existen muchas plántulas (3800 individuos/ha) que no llegan a establecerse por la competencia de los árboles maduros (Ayma-Romay et al. 2007). En cada parcela se plantaron diez plántulas en hoyos de 20x20 $\mathrm{cm}$ ubicados en micrositios distintos en cuanto a tipos de cobertura vegetal, piedras y pendiente, dentro de lo posible (estimación visual). Las plántulas no recibieron riego. La plantación se realizó en verano (temporada de lluvia). Durante los dos primeros años, las parcelas estuvieron clausuradas con alambre de púas para evitar el ingreso del ganado; desde el tercer año fueron abiertas a solicitud de la comunidad, lo que no tuvo mayores consecuencias para el estudio porque todas tuvieron el mismo tratamiento y los disturbios fueron muy bajos (hubo muy bajas señales de ganadería en las parcelas). En general, las plántulas muertas no mostraron signos de ramoneo, pero sí de deshidratación, aunque esto no fue evaluado.

\section{Evaluación de campo}

Las mediciones se realizaron a los siete (septiembre de 2009), catorce (abril de 2010) y 51 meses (abril de 2013) después de la plantación. Para caracterizar el micrositio alrededor de cada plántula reforestada se evaluó visualmente la cobertura (\%) de musgos, arbustos y piedras, pastos, hierbas y suelo descubierto en un área de $1 \mathrm{~m}$ de radio. También se midió el valor de la pendiente predominante (\%). El promedio entre fechas de estas mediciones se usó para los análisis estadísticos.

La sobrevivencia final se registró como planta viva o muerta (planta viva $=1$; planta muerta=0) al final del experimento. El crecimiento se midió como la diferencia entre fechas de la altura de la plántula desde el cuello del tallo hasta el ápice de la rama más larga y dominante. La suma de los incrementos hasta el final del experimento o hasta la muerte de cada plántula fue el crecimiento acumulado.

\section{Análisis de datos}

La variación de cada uno de los componentes del micrositio entre las cinco altitudes fue analizada con la prueba de Kruskal-Wallis. Posteriormente, para analizar la influencia de la altitud (factor fijo) y la parcela (factor anidado) sobre el porcentaje de sobrevivencia final y el crecimiento acumulado de las 
plántulas se emplearon modelos lineales generalizados mixtos (MLGM), de la familia binomial (enlace logístico) y de la familia gaussiana (enlace identidad), respectivamente. No se incluyeron los componentes del micrositio como covariables en este análisis porque estuvieron muy correlacionados con la altitud. Para ver específicamente qué altitudes se diferenciaban en cuanto a sobrevivencia y crecimiento, se realizó posteriormente una prueba de comparación múltiple de medias de Tukey entre altitudes para los MLGM con el paquete "multcomp" (Hothorn et al. 2008). Más adelante se analizó la influencia de cada uno de los componentes del micrositio sobre la sobrevivencia y el crecimiento acumulado de las plántulas, para cada altitud por separado, a través de MLGM. Este análisis incluyó a las parcelas repetidas en cada altitud como factor aleatorio y evitó confundir el efecto de la altitud y de los componentes del micrositio. La variable de crecimiento acumulado fue transformada a su logaritmo con base diez y las variables de micrositio con el arcoseno de su raíz cuadrada para cumplir supuestos de homocedasticidad y normalidad. El análisis fue realizado en R project ( $R$ Core Team 2015). Los paquetes utilizados para el análisis con los MLGM fueron: "nlme" (Pinheiro et al. 2016) y "MASS" (Venables and Ripley 2002).

\section{Resultados}

\section{Características del micrositio}

La cobertura de los componentes bióticos y abióticos del micrositio de las plántulas reforestadas varió significativamente con la altitud (Tabla 1). La cobertura de pastos fue dominante en todas las altitudes, sobre todo en altitudes mayores, seguido de las hierbas, que ocuparon regularmente todas las altitudes, con excepción de la más alta y la intermedia (donde su cobertura fue menor). La cobertura de musgos, piedras (de un diámetro aproximado entre 2 y $30 \mathrm{~cm}$ ) y de arbustos (de 30 a $150 \mathrm{~cm}$ de alto) tuvo una dominancia menor; además, estas coberturas fueron mayores en altitudes inferiores. El porcentaje de suelo descubierto fue muy escaso, pero con mayor proporción en la altitud intermedia. La pendiente fue leve en todas las altitudes, excepto a 3590 m s.n.m., donde hubo pendientes mayores (Tabla 1).

\section{Sobrevivencia}

La sobrevivencia final de las plántulas varió entre 0 y $23 \%$, y fue influida por la altitud (Tabla 2). La sobrevivencia fue mayor en la altitud intermedia y disminuyó hacia altitudes menores y mayores (patrón unimodal). Por ejemplo, en la altitud mayor no sobrevivió ninguna plántula (Figura 1). Los componentes abióticos y bióticos del micrositio, analizados por separado y para cada altitud, no mostraron influencia significativa sobre la sobrevivencia.

\section{Crecimiento}

El crecimiento acumulado promedio de todas las plántulas fue de $2.1 \mathrm{~cm}$, y tuvo un rango amplio de valores (mínimo $=0 \mathrm{~cm}$ y máximo $=41 \mathrm{~cm}$ ). El crecimiento también fue influido por la altitud (Tabla 3). Sin embargo, a diferencia del patrón unimodal de la sobrevivencia, el crecimiento fue mayor en el rango de las altitudes menores e intermedias (entre 2747 y 3300 m s.n.m.) y fue menor en altitudes mayores o iguales a 3590 m s.n.m. (Figura 2).

La cobertura de arbustos, piedras y el porcentaje de pendiente modificaron el efecto de la altitud sobre el crecimiento en algunas altitudes. La cobertura de arbustos

Tabla 1. Caracterización de la cobertura de componentes abióticos y bióticos del micrositio por altitudes (\%). Se muestra la mediana, valor mínimo y máximo entre paréntesis. Las diferencias significativas entre altitudes se evaluaron con la prueba de suma de rangos de Kruskal-Wallis (las diferentes letras muestran esas diferencias).

Table 1. Description of abiotic and biotic components of the microsites at different altitudes (\%). The median, maximum and minimum values are shown in brackets. The Kruskal-Wallis test was used to estimate statistical differences among altitudes (different letters shows these differences).

\begin{tabular}{lcccccr}
\hline $\begin{array}{c}\text { Componentes } \\
(\%)\end{array}$ & \multicolumn{5}{c}{ Altitud (m s.n.m.) } \\
\hline Arbustos & 11(5-39)A & 18(7-39)B & 6(1-11)C & 3(0-19)C & $0.2(0-6) \mathrm{D}$ & $<$ \\
Hierbas & 16(9-32)A & 22(9-48)B & 13(5-29)C & 16(7-41)A & $10(6-28) \mathrm{C}$ & $<0.0001$ \\
Musgos & 12(2-26)A & 13(6-22)A & 11(5-20)A & $10(4-23) \mathrm{A}$ & $5(1-13) \mathrm{B}$ & $<0.0001$ \\
Pastos & 42(17-59)A & 33(7-57)A & 55(39-71)B & $56(28-72) \mathrm{B}$ & $73(56-85) \mathrm{C}$ & $<0.0001$ \\
Piedras & 10(1-53)A & 4(0-17)B & 4(0-17)B & 3(0-13)BC & $3(0-8) \mathrm{C}$ & $<0.0001$ \\
Suelo & 1(0-6)A & 1(0-5)A & 2(0-10)B & 0.5(0-3)A & $1(0-3) \mathrm{A}$ & 0.002 \\
Pendiente & 3(0-16)A & 5(2-7)A & 5(0-10)A & 8(2-16)B & $4(0-10) \mathrm{A}$ & $<0.0001$ \\
\hline
\end{tabular}


Tabla 2. Efecto de la altitud sobre la sobrevivencia final de plántulas de $P$. glomeratus reforestadas en pastizales entre 2747 y 3850 m s.n.m. El análisis fue realizado con el ajuste de un modelo lineal generalizado mixto, que incluyó las parcelas repetidas de cada altitud como factor anidado.

Table 2. Effect of altitude on the seedling survival of $P$. glomeratus reforested in tussock grasslands among 2747 and $3850 \mathrm{~m}$ a.s.l. The analysis was made using a generalized linear mixed-effects model, which included the repeated plots of each altitude as a nested factor.

\begin{tabular}{ccc}
\hline $\begin{array}{c}\text { Altitud } \\
\text { (m s.n.m.) }\end{array}$ & Coeficiente & $P$ \\
\hline 2747 & -2.19 & 0.0001 \\
3005 & -0.44 & 0.61 \\
3300 & 1.0 & 0.14 \\
3590 & -1.17 & 0.27 \\
3850 & -27.36 & 0.99 \\
\hline
\end{tabular}

Tabla 3. Efecto de la altitud sobre el crecimiento acumulado de plántulas de $P$. glomeratus reforestadas en pastizales entre 2747 y 3850 m s.n.m. El análisis fue realizado con el ajuste de un modelo linear generalizado mixto, que incluyó las parcelas repetidas de cada altitud como factor anidado.

Table 3. Effect of altitude on the cumulative seedling growth of $P$. glomeratus, which was reforested in tussock grasslands among 2747 and $3850 \mathrm{~m}$ a.s.l. The analysis was made using a generalized linear mixed-effects model, which included the repeated plots of each altitude as a nested factor.

\begin{tabular}{ccc}
\hline $\begin{array}{c}\text { Altitud } \\
\text { (m s.n.m.) }\end{array}$ & Coeficiente & $P$ \\
\hline 2747 & 0.50 & $<0.001$ \\
3005 & -0.10 & 0.12 \\
3300 & -0.06 & 0.31 \\
3590 & -0.33 & $<0.001$ \\
3850 & -0.36 & $<0.001$ \\
\hline
\end{tabular}

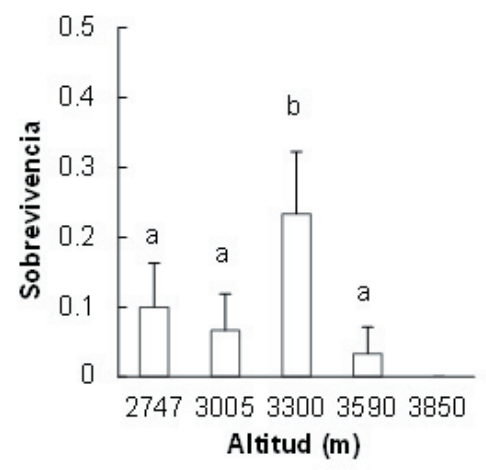

Figura 1. Sobrevivencia (estimada como proporción) de plántulas de P. glomeratus reforestadas en pastizales a lo largo de diferentes altitudes y evaluada después de 51 meses. Las barras muestran el error estándar. Letras distintas muestran diferencias significativas entre altitudes $(\mathrm{P}<0.05)$.

Figure 1. Seedling survival (expressed as proportions) of P. glomeratus which were reforested in tussock grasslands along different altitudes, and analyzed after 51 months. The bars show the standard error. Different letters indicate significative statistical differences among altitudes $(\mathrm{P}<0.05)$.

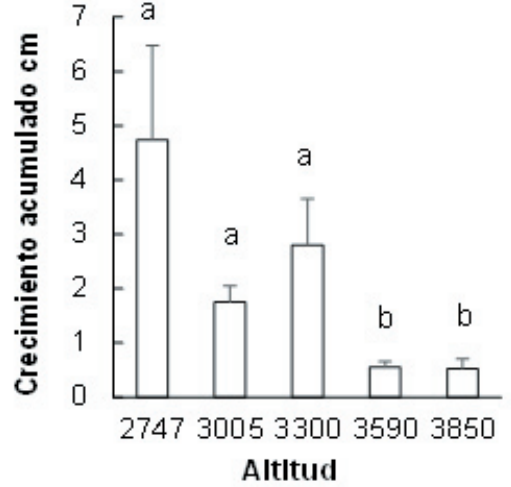

Figura 2. Crecimiento acumulado promedio de las plántulas de $P$. glomeratus reforestadas en pastizales a lo largo de diferentes altitudes y evaluado después de 51 meses. Las barras muestran el error estándar. Letras distintas muestran diferencias significativas entre altitudes $(P<0.05)$.

Figure 2. Cumulative growth of $P$. glomeratus seedlings, which were reforested in tussock grasslands along different altitudes and analyzed after of 51 months. The bars show the standard error. Different letters indicate significant statistical differences among altitudes $(P<0.05)$.

incrementó significativamente el crecimiento de las plántulas en la altitud inferior $(P=0.05$; $\mathrm{n}=30$ ) (Figura 3). La cobertura de piedras redujo significativamente el crecimiento de las plantas a 3300 m s.n.m. $(P=0.05 ; \mathrm{n}=30)$ (Figura 4), pero la aumentó significativamente $(P=0.04 ; \mathrm{n}=30)$ (Figura 5) a 3590 m s.n.m. A esta altitud, la mayor pendiente también aumentó el crecimiento $(P=0.02 ; \mathrm{n}=30)$ (Figura 6; Tabla 1). Los otros componentes del micrositio no mostraron influencia significativa sobre el crecimiento.

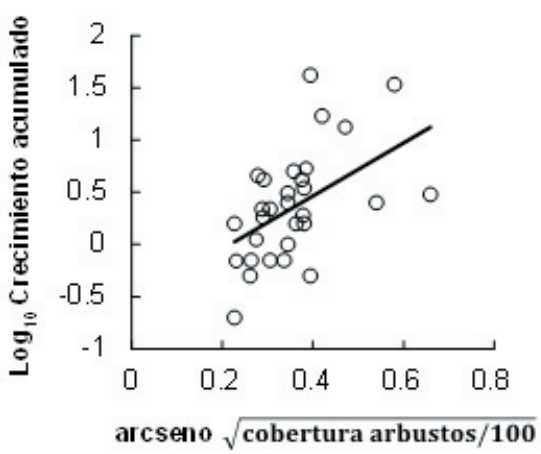

Figura 3. Efecto positivo de la cobertura de arbustos sobre el crecimiento acumulado de plántulas de $P$. glomeratus reforestadas en pastizales en la altitud inferior $(2747 \mathrm{~m}$ s.n.m.), evaluado después de 51 meses de la plantación.

Figure 3. Positive effect of the shrub cover on the cumulative growth of $P$. glomeratus seedlings, which were reforested in tussock grasslands at the lowest altitude ( 2747 $\mathrm{m}$ a.s.l.). The plot shows data evaluated after 51 months of the plantation. 


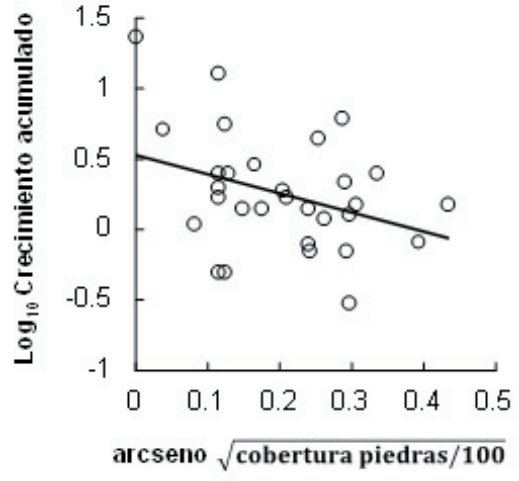

Figura 4. Efecto negativo de la cobertura de piedras sobre el crecimiento acumulado de plántulas de $P$. glomeratus reforestadas en pastizales en la altitud intermedia de 3300 m s.n.m., evaluado después de 51 meses de la reforestación.

Figure 4. Negative effect of the rock outcrop cover on the cumulative growth of $P$. glomeratus seedlings, which were reforested in tussock grasslands at an intermediate altitude of $3300 \mathrm{~m}$ a.s.l. The analysis shows data evaluated after 51 months of the plantation.

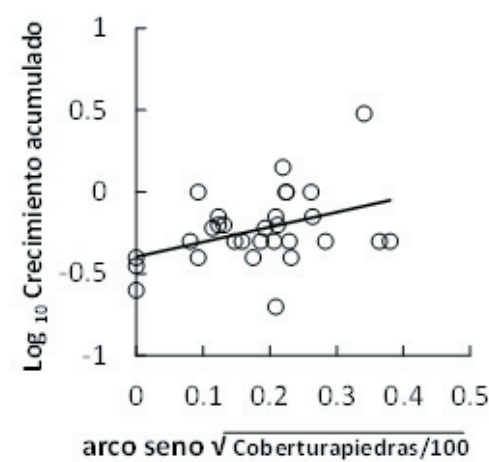

Figura 5. Efecto positivo de la cobertura de piedras sobre el crecimiento acumulado de plántulas de $P$. glomeratus, reforestadas en pastizales a 3590 m s.n.m., evaluado después de 51 meses de la plantación.

Figure 5. Positive effect of rock outcrop cover percentage on the cumulative growth of $P$. glomeratus seedlings, which were reforested in tussock grasslands at $3590 \mathrm{~m}$ a.s.l. The figure shows data evaluated after of 51 months of the plantation.

\section{DisCUSIÓN}

\section{Efecto de la altitud y el micrositio}

La sobrevivencia de plántulas reforestadas de $P$. glomeratus fue controlada por la altitud, y el crecimiento por la altitud y algunos componentes del micrositio. El patrón unimodal de la sobrevivencia respecto a la altitud generalmente es causado por el estrés ambiental en altitudes mayores (i.e., frio, alta radiación) y en altitudes menores (i.e., calor y déficit hídrico); así las condiciones ambientales más favorables se dan en altitudes intermedias,

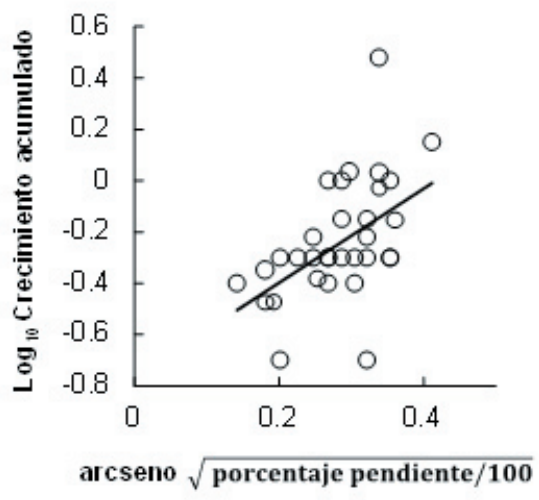

Figura 6. Efecto positivo del porcentaje de pendiente sobre el crecimiento acumulado de plántulas de $P$. glomeratus reforestadas en pastizales a una altitud de $3590 \mathrm{~m}$ s.n.m., evaluado después de 51 meses de la plantación.

Figure 6. Positive effect of the slope percentage on the cumulative growth of $P$. glomeratus seedlings, which were reforested in tussock grasslands at $3590 \mathrm{~m}$ a.s.l. The analysis shows data evaluated after 51 months of the plantation.

tal como se encontró en otros estudios realizados en gradientes altitudinales (Körner and Paulsen 2004; Smith et al. 2009; Marcora et al. 2013). Los componentes evaluados del micrositio a través del gradiente altitudinal no mostraron un efecto significativo sobre la sobrevivencia de las plántulas porque posiblemente no modificaron las condiciones microambientales tanto como para evitar la mortalidad.

El mayor crecimiento de las plántulas entre las altitudes menores y la intermedia (de 2747 a 3300 m s.n.m.) puede deberse a que algunos componentes abióticos o bióticos del micrositio modificaron el efecto de la altitud (Bertness and Callaway 1994). En la altitud inferior, la cobertura de arbustos incrementó el crecimiento de las plántulas, mientras que en la altitud intermedia, la cobertura de piedras la disminuyó. Ambos factores posiblemente igualaron el crecimiento entre esas altitudes.

El menor crecimiento de las plántulas en altitudes $>3600$ m s.n.m. probablemente se debió a las bajas temperaturas en la noche y a la alta radiación (Báez et al. 2011), las que no son evitadas de manera importante por ninguno de los micrositios evaluados. Por ejemplo, las plantas de Podocarpusoleifoliusen altitudes iguales o mayores a $3200 \mathrm{~m}$ s.n.m. son susceptibles a congelamiento con temperaturas inferiores a $-3^{\circ} \mathrm{C}$. Además, reducen sus tasas de fotosíntesis y aumentan las de respiración para mantener su funcionamiento y reparar los daños (Cavieres et al. 2000). 


\section{Efectodeloscomponentesbióticosyabióticosdel micrositio}

Los arbustos en la altitud inferior podrían haber reducido el estrés ambiental para las plántulas de $P$. glomeratus porque dieron sombra y aminoraron la deshidratación en condiciones ambientales con mayor radiación solar, déficit hídrico y temperatura (Bertness and Callaway 1994; Anthelme et al. 2014a). En altitudes mayores, este efecto fue menos importante para las plántulas porque la cobertura de los arbustos fue cada vez menor con el aumento de la altitud (Tabla 1) y porque la sombra de los arbustos probablemente haya sido menos beneficiosa para las plántulas en altitudes donde el déficit hídrico y la temperatura elevada tiene menos importancia (Zamora et al. 2004; Padilla and Pugnaire 2006; Maestre et al. 2009). Sin embargo, es importante estudiar con mayor detalle el efecto nodriza de los arbustos en altas altitudes para reducir alta radiación sobre las plantas (Báez et al. 2011).

A los 3590 m s.n.m., la pendiente influyó en el crecimiento de las plantas posiblemente porque redujo las bajas temperaturas propias de esa altitud (en particular durante el invierno) (Simpson 1986; Doust et al. 2006). Sin embargo, este factor no fue significativo en otras altitudes porque la pendiente tuvo un menor rango de valores (Tabla 1) y porque la protección contra las bajas temperaturas ya no fue tan importante en altitudes menores. Por otro lado, la pendiente también pudo tener un efecto positivo en esta altitud porque redujo el daño de las plantas por el ganado vacuno presente en altas altitudes de la comunidad (observación personal) y que pudo afectar desde el tercer año. Por ejemplo, se ha observado que el ganado vacuno evita recorrer pendientes más pronunciadas (Fjeldså and Kessler 2004).

La cobertura de piedras tuvo un efecto negativo sobre el crecimiento en la altitud intermedia, posiblemente porque se asoció con una mayor degradación del suelo (en esta altitud hubo más suelo descubierto [Tabla 1]). Por ejemplo, en otro estudio realizado en pastizales con severa degradación, las piedras también estuvieron asociadas a un menor crecimiento y sobrevivencia de plántulas de Polylepis australis (Renison et al. 2004). Por otro lado, el efecto positivo de las piedras en una altitud mayor se debió a que los suelos estuvieron menos degradados en esta altitud y a que las piedras acumulan calor en el día y lo irradian durante la noche, característica relevante en altitudes mayores (Körner and Paulsen 2004; Peters et al. 2008).

La cobertura de musgos y hierbas no tuvo efecto sobre el crecimiento y la sobrevivencia en ninguna altitud porque su cobertura fue poco estable durante los años (en época seca fue reducida y en época húmeda, alta) (observación personal). De similar manera, la cobertura de pastos, pese a su dominancia, tampoco proporcionó mejor microambiente para las plántulas. Se ha observado que, en general, los pastos no aminoran las altas oscilaciones de temperatura y radiación, características de los ecosistemas de pastizales en los Andes (Navarro 2005). Sin embargo, en otros estudios se mostró que los musgos (en ambientes húmedos) (Rovere and Calabrese 2011) y los pastos (en ambientes áridos de los Andes) tuvieron un efecto positivo sobre el establecimiento de árboles latifoliados (Anthelme et al. 2014b).

\section{Recomendaciones para la reforestación de $P$. glomeratus}

Las plántulas de $P$.glomeratus reforestadas en pastizales no se establecen satisfactoriamente en altitudes $>3600$ m s.n.m., aunque es importante probar el efecto de la pendiente y las piedras como facilitadores en estas altitudes. La reforestación, puede realizarse de manera satisfactoria en altitudes intermedias (3300 m s.n.m), donde predominan los bosques maduros de la especie (Navarro 2005), y puede desplazarse hacia altitudes menores (hasta 2500 m s.n.m) donde los bosques fueron convertidos a cultivos y pastizales. Es importante probar el efecto positivo de los arbustos en altitudes menores a 3300 m s.n.m. porque pueden ser facilitadores importantes para el establecimiento de la especie (Zamora et al. 2004; Báez et al. 2011).

La tasa de sobrevivencia y crecimiento de las plántulas de $P$. glomeratus son bajas en comparación con las de otras especies nativas de Podocarpus (Bergin and Kimberley 2014) o con exóticas de rápido crecimiento (Fjeldså and Kessler 2004). Sin embargo, identificar el micrositio y la altitud adecuada puede mejorar el establecimiento de plántulas. Asimismo, debido a que en el estudio se usaron plántulas del bosque, es posible que plántulas criadas en vivero, más vigorosas y con mayor tamaño, obtengan mayor tasa de sobrevivencia y crecimiento. 
Agradecimientos. A la comunidad de Pajchanti por colaborar y permitirnos realizar los experimentos en su territorio. También a la Foundation Rufford Small Grants que financió nuestras actividades de reforestación, a la editora Ana M. Cingolani y a los revisores anónimos de la revista por sus gentiles y acertadas recomendaciones.

\section{REFERENCIAS}

Anthelme, F., L. Cavieres, and O. Dangles. 2014a. Facilitation among plants in alpine environments in the face of climate change. Front Plant Sci 387(5):1-15.

Anthelme, F., L. Gómez-Aparicio, and R. Montúfar. 2014b. Nurse-based restoration of degraded tropical forests with tussock grasses: experimental support from the Andean cloud forest. J Appl Ecol 51:1534-1543.

Ayma-Romay, A. I., and E. S. Sanzetenea. 2008. Variaciones fenológicas de especies de Podocarpaceae en estación seca de los Yungas (Cochabamba, Bolivia). Ecología en Bolivia 43(1):16-28.

Ayma-Romay, A. I., E. Padilla, and E. Calani. 2007. Estructura, composición y regeneración de un bosque de neblina: sugerencias silviculturales para Podocarpus glomeratus (Podocarpaceae) en la comunidad de Pajchanti (Cochabamba, Bolivia). Revista Boliviana de Ecología y Conservación Ambiental 21:27-42.

Ayma-Romay, A. I., and E. Padilla. 2009. Efecto de la tala de Podocarpus glomeratus (Podocarpaceae) sobre la estructura de un bosque de neblina en los Andes (Cochabamba, Bolivia). Rev Peru Biol 16(1):73-79.

Báez, S., F. Cuesta, Y. Cáceres, C. Arnillas, and R. Vásquez. 2011. Síntesis del conocimiento de los efectos del cambio climático en la biodiversidad de los Andes tropicales. CONDESAN, Lima.

Beniston, M. 2003. Climatic change in mountain regions: A review of possible impacts. Climatic Change 59:5-31.

Bergin, D., and M. Kimberley. 2014. Factors influencing natural regeneration of totara (Podocarpus totara D. Don) on grazed hill country grassland in Northland, New Zealand. New Zeal J For Sci 44:1-13.

Bertness, M. D., and R. Callaway. 1994. Positive interactions in communities. Trends Ecol Evol 9:191-193.

Cavieres, L., F. Rada, A. Azócar, C. García-Núñez, and H. Cabrera. 2000. Gas exchange and low temperature resistance in two tropical high mountain tree species from the Venezuelan Andes. Acta Oecologica 21(3):203-211

Cernusak, L. A., H. Adie, P. Bellingham, E. Biffin, T. Brodribb, D. Coomes; et al. 2011. Podocarpaceae in tropical forests: a synthesis. Pp. 189-195 en: B. Turner and L. Cernusak (eds.). Ecology of the Podocarpaceae in Tropical Forests. Smithsonian Institution Scholarly Press. Washington.

Cierjacks, A., K. Wesche, and I. Hensen. 2007. Potential lateral expansion of Polylepis forest fragments in central Ecuador. Forest Ecol Manag 242:477-486.

Dalling, J., P. Barkan, P. J. Bellingham, J. Healey, E. Tanner, and J. Murillo. 2011. Ecology and distribution of neotropical Podocarpaceae. Pp. 43 -56 en: B. Turner and L. Cernusak (eds.). Ecology of the Podocarpaceae in Tropical Forests. Smithsonian Institution Scholarly Press, Washington.

Doust, S. J., P. Erskine, and D. Lamb. 2006. Direct seeding to restore rainforest species: microsite effects on the early establishment and growth of rainforest tree seedlings on degraded land in the wet tropics of Australia. Forest Ecol Manag 234:333-343.

Fjeldså, J., and M. Kessler. 2004. Conservación de la biodiversidad de los bosques de Polylepis de las tierras altas de Bolivia. Editorial FAN, Santa Cruz.

Grubb, P. J. 1977. The maintenance of species-richness in plant communities: the importance of the regeneration niche. Biol Rev 52:107-145.

Hothorn, T., F. Bretz, and P. Westfall. 2008. Simultaneous inference in general parametric models. Biometrical Journal 50:346-363.

Körner, C., and J. Paulsen. 2004. A world-wide study of high altitude treeline temperatures. J Biogeogr 31:713-732.

Marcora, P., D. Renison, A. I. País-Bosch, M. R. Cabido, and P. A. Tecco. 2013. The effect of altitude and grazing on seedling establishment of woody species in central Argentina. Forest Ecol Manag 291:300-307.

Maestre, F. T., R. Callaway, F. Valladares, and C. Lortie. 2009. Refining the stress-gradient hypothesis for competition and facilitation in plant communities. J Ecol 97:199-205.

Navarro, G. 2005. Provincia biogeográfica de los yungas. Pp. 279-339 en: G. Navarro and M. Maldonado (eds.). Geografía ecológica de Bolivia. Centro de Ecología y Difusión Simón I. Patiño, Santa Cruz.

Padilla, F. M., and F. Pugnaire. 2006. The role of nurse plants in the restoration of degraded environments. Front Ecol Environ 4:196-202.

Peters, E., C. Martorell, and E. Ezcurra. 2008. Nurse rocks are more important than nurse plants in determining the distribution and establishment of Globose cacti (Mammillaria) in the Tehuacán Valley, Mexico. J Arid Environ 72: 593-601.

Pinheiro, J., D. Bates, S. Debroy, and D. Sarkar. 2016. nlme: linear and nonlinear mixed effects models. URL: http: / /CRAN.R-project.org/package=nlme.

R Core Team. 2015. R: a language and environment for statistical computing. R Foundation for Statistical Computing, Vienna. http://www.r-project.org.

Renison, D., I. Hensen, and A. Cingolani. 2004. Anthropogenic soil degradation affects seed viability in Polylepis australis mountain forests of central Argentina. Forest Ecol Manag 196:327-333.

Rovere, A., and G. Calabrese. 2011. Diversidad de musgos en ambientes degradados sujetos a restauración en el Parque 
Nacional Lago Puelo. Rev Chil Hist Nat 84:571-580.

Simpson, B. 1986. Speciation and specialization of Polylepis in the Andes. Pp. 304 -316 en: F. Vuillemier and M. Monasterio (eds.). High altitude tropical biogeography. Oxford University Press, Nueva York.

Smith, W., M. Germino, D. Johnson, and K. Reinhardt. 2009. The altitude of alpine treeline: A bellwether of climate change effects. Bot. Rev 75:163-190.

Venables, W., and B. Ripley. 2002. Modern applied statistics with S. Springer, New York.

Zamora, R., P. García-Fayos, and L. Gómez-Aparicio. 2004. Las interacciones planta-planta y planta animal en el contexto de la sucesión ecológica. Pp. 371-393 en: F. Valladares (ed.). Ecología del bosque mediterráneo en un mundo cambiante. EGRAF, Madrid. 